\title{
J055025
}

\section{Measurement of microparticle distribution in microchannel flow by using process tomography}

\author{
Nur Tantiyani ALI OTHMAN* and Masahiro TAKEI
}

Graduate School of Mechanical Engineering, Artificial System Science Division, Chiba University, Japan

In the present study, a cross-sectional capacitance is measured in a microchannel with 12 multi-layered electrodes at upstream and downstream cross-section. Based on the cross-sectional capacitance measurement, the particle volume fraction is determined at two configuration areas of electrode pair; at horizontal and vertical pair. The particle volume fraction shows some increment $9.3 \%$ at electrode pair near the walls, whilst decreased $0.96 \%$ at center electrode pair from upstream to downstream cross-section. As position move from inlets to outlets area, the particles drift away from center streamlines to near walls and a number of particles migrations near the walls are clearly increased at outlet area.

Key Words : Microchanel, tomography, cross-section, multi-phase flow

\section{Introduction}

Recently, micro-electro-mechanical-systems (MEMS) technology is growing rapidly, shows a remarkable developed and widely used in micro-analytical measurement and micro bio-medical field (1-3). In a field of miniaturization devices, mixing processes are first investigated and important issue at microscale to ensure homogenization of material in solutions. Generally, particles migration is controlled by hydrodynamic forces and Brownian diffusion. However, turbulent flows cannot be generated when the dimensions of the structure are less than hundreds of micrometers. Therefore, the particle does not remain following a fluid streamlines and particles migration is frequently observed (4-5). A neutrally buoyant particle in the microchannel is known to undergo a radial migration for finite conditions flow. Many studied of the particles migration in the microscale are reported (6-12). Their studied show the particles migration induced by inertial lifts forces, which affect particles advection and particle interactions to move from center streamlines to others areas. Although the particles migration induced by the inertial lift forces in the microscale is a unique motion but the detail studied on it is still required and not fully understood. Therefore, the purpose of this study is to measure the particle distribution and more particularly to study the behavior of particle migration from the upstream to downstream cross-section. The diamond shape of the cross-sectional microchannel is captured using high speed micro computed tomography as shows in figure 1 (a-b). Figure 1(c) shows a schematic of cross-sectional microchannel with height $800 \mu \mathrm{m}$ in $y$-axis and width $700 \mu \mathrm{m}$ in $x$-axis. Each cross-section is embedding with 12 multi-layered electrodes and the sensor electrode is connected to electrical tomography system.

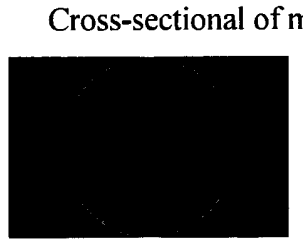

(a)

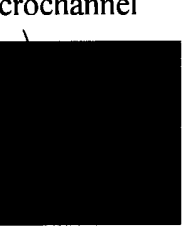

(b)



(c)

Figure 1 Cross-sectional microchannel and its dimension.

- Division of Artificial System Science, Chiba University, 1-33 Yayoi, Inage, 263-8522, Chiba Japan

E-mail: tantiyani@yahoo.com 


\section{Results and discussion}

Figure 2 show a comparison of the particle distribution at vertical and horizontal pair measurement, respectively for $1.5 \mu \mathrm{m}$ particles at cross-section I, III and V. At cross-section I and III, the particle volume fraction shows some increment from electrode pairs near the wall (electrode 6-7 and 1-12) to electrode pairs at the center microchannel (electrode 4-9 and 3-10). At the cross-section III, the particle volume fraction distribution is slowly decreased at the center and gradually increased at near the wall. The particle volume fraction at electrode pair 4-9 slightly $23.1 \%$ decreased from cross-section I to cross-section III. It shows particles are slowly transported and migrated along the streamlines from the centerlines to near the wall. While, at cross-section V, the pattern of the particle volume fraction is in an inverse shape with the result at cross-section I and III. The higher on the particle volume fraction is indicated at the electrode pairs near the walls (electrode 4-9 and 3-10).

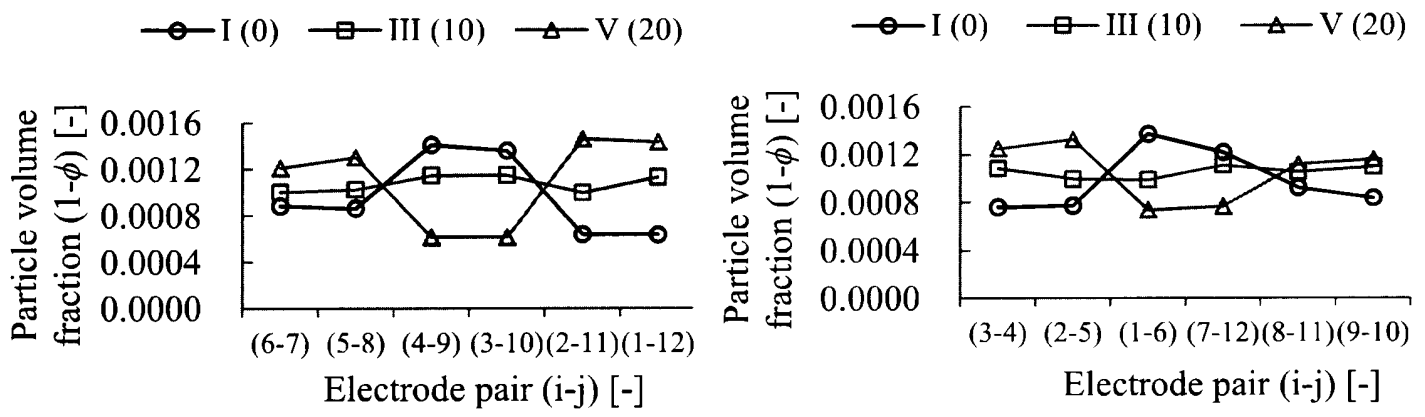

Figure 2 Comparison of particle volume fraction at cross-section I, III and V

The same result on particle volume fraction is indicated at horizontal pair where greater of particle volume fraction indicated at center electrode pair; at electrode pairs 1-6 and 7-12 and the lowest at electrode pair 3-4 and 9-10; near the walls. It is because in the vicinity of solid walls flow is not uniform. This causes perturbations in the force experienced by the particles. In microsystem, it is often the case that the size of particles is comparable to the size of channels in where particles are contained. Therefore, particles seldom experience uniform flow fields. Overall, the particle volume fraction is increased gradually from upstream to downstream cross-section in both electrode pair areas measurement. The electrode pair nearest to center streamlines shows about $37.2 \%$ decreased of the particle volume fraction than the others electrode pair near the walls.

\section{Conclusion}

This paper has investigated the measurement of particle volume fraction distribution based on the cross-sectional capacitance measurement at upstream and downstream cross-section. Particles are distributed non-uniformly due to particles migration induced by inertial lift forces and gradient velocity profile. The particle volume fraction at downstream cross-section is $9.3 \%$ increased at electrode pair near the walls and slightly decreased $0.96 \%$ at electrode pair near the center streamlines and the particle volume fraction reveals that particles migrate near the walls from the upstream to the downstream cross-sections.

\section{Acknowledgements}

This study is supported by Adaptable and Seamless Technology Transfer Program through Target-driven R\&D (A-Step) of Japan Science and Technology Agency (\#AS2311054B).

\section{References}

(1) G. K., Ananthasuresh and S. Chakraborty, "Micromechanics of engineered and biological systems", Current Trends in Science: Platinum Jubilee Special, (2010). 
(2) J. Feng and D. D. Joseph, "The motion of particles suspended in viscoelastic under torsional shear", J. Fluid Mech., Vol. 324, (1996), pp. 199-222.

(3) Y. W. Kim and J. Y. Yo, "The lateral migration of neutrally-buoyant spheres transported through square microchannels", Journal of Micromechanics and Microengineering, Vol. 18, 065015, (2008).

(4) G. Segre and A. Silberberg, "Behavior of macroscopic rigid spheres in Poiseuille flow: Part 1. Determination of local concentration by statistical analysis of particle passage through crossed light beams", J. Fluid Mech., Vol. 14, (1962), pp. 115-135.

(5) R. G. Cox and S. G. Mason, "Suspended particles in fluid flow through tubes", Ann. Rev. Fluid Mech. Vol. 3, (1971), pp. 291-316.

(6) D. R. Oliver, "Influence of particle rotation on radial migration in the Poiseuille flow of suspensions", Journal Nature, Vol. 194, 1269-71, (1962).

(7) H. L. Goldsmith and S. G Mason, "The flow of suspensions through tubes: I. Single spheres, rods, and discs", J. Colloid Sci, 17 448-76, (1962).

(8) R. C. Jeffrey and J. R. A. Pearson, "Particle motion in laminar vertical tube flow", J. Fluid Mech, 22 721-35, (1965).

(9) B. Chun and A. J. C. Ladd, "Inertial migration of neutrally buoyant particles in a square duct: An investigation of multiple equilibrium positions", Phys. Fluids, Vol. 18, 03170, (2006).

(10) B. P. Ho and L. G Leal, "Inertial migration of rigid spheres in two-dimensional unidirectional flows", J. Fluid Mech, Vol. 65, (1974), pp. 365-400.

(11) J. Feng, H. H. Hu and D. D. Joseph, "Direct simulation of initial value problems for the motion of solid bodies in a Newtonian fluid: Part 2. Couette and Poiseuille flows", J. Fluid Mech, 277 271-30, (1994).

(12) A. Asgar, S. Bhagal, S. Sathyakumar, Kuntaegowdanahaili, and I. Papautsky, "Enhanced particle filtration in straight microchannel using shear-modulated inertial migration", Physics of Fluids, 101702, (2008). 